\title{
Features of Forecasting Reliability of 6-10 kV Overhead Electric Lines According to Statistics of Their Failures and Reconditionings
}

\author{
Basmanov V.G., Kholmanskikh V.M. \\ Vyatka State University \\ Kirov, Russian Federation
}

\begin{abstract}
This work is aimed at forecasting justification of the failure time of the $6-10 \mathrm{kV}$ overhead electric lines (OEL) during the normalized period in its operation based on comparison with the statistics of failures and reconditionings on the previous intervals with the use of the OEL availability function, statistical availability coefficient, normalized forecasting interval and the accepted values of the availability coefficient on the forecasting interval. To achieve the goal set the OEL is described as an object of a multiple action, its failure and reconditioning flows are accepted as the simplest Poisson, and for the theoretical analysis of the variation character in the availability coefficient, the probability theory methods along with a mass service were used. The most significant result is justification of the use for the forecasting of the OEL failure time of a new convenient exponential expression of its availability function on the normalized period of time being forecasted. Unlike the accepted in the theory of reliability the availability function with two parameters $T$ and $T_{\text {r.av }}$ (average times of work and reconditionings), the proposed expression uses one parameter of distribution $T_{v i r}$ (virtual nonfailure operating time). The significance of the results obtained consists in that controlling the dynamics of the variation in the statistical coefficient of availability of the OEL on the previous time intervals makes it possible to forecast its failure time during the forthcoming normalized periods of operation.
\end{abstract}

Keywords: overhead lines, coefficient of technical availability, forecasting normalized interval, virtual non-failure operating time, confidence probability.

DOI: https://doi.org/10.52254/1857-0070.2021.4-52.01

UDC: 621.315 .17

Particularități ale prognozei fiabilității liniilor electrice aeriene de 6-10 kV

din datele statistice privind refuzurile și restabilirile

Basmanov V.G., Kholmanskikh V.M.

Universitatea de Stat din Viatka

Kirov, Federația Rusă

Rezumat. Scopul lucrării este de a justifica prognoza timpului de întrerupere a liniilor electrice aeriene (LEA) de 6-10 kV pentru intervalul normat de exploatare a acestora în baza comparării datelor statistice privind refuzurile și restabilirile pentru intervalele anterioare, utilizând funcția de disponibilitate pentru LEA, factorul de disponibilitate statistică, intervalul normat de prognoză și valorile admisibile ale factorului de disponibilitate în intervalul de prognoză. Pentru atingerea obiectivului, linia aeriană a fost considerată ca un obiect cu acțiuni repetate, fluxurile de refuzuri și restabiliri au fost considerate cele mai simple fluxuri Poisson, iar pentru analiza teoretică a caracterului de variație a factorului de disponibilitate au fost utilizate metodele probabilităților și a teoriilor de deservire în masă. Eroarea calculelor la așa o substituire chiar în diapazonul variației valorii coeficientului de disponibilitate pentru anul calendaristic, de la 0,999 până la 0,995 constituie de la 0,018 până la 0,369\%. Rezultatul cel mai important este o justificare a aplicării unei noi expresii exponențiale potrivite a funcției de disponibilitate la intervalul de timp normat de prognoză pentru prognozarea timpului de de deconectare a LEA. Spre deosebire de funcția de disponibilitate cu doi parametri (timpul mediu de funcționare și de restabilire) acceptată în teoria fiabilității, expresia propusă pentru funcția de disponibilitate a liniilor aeriene utilizează un singur parametru de distribuție $T_{v i r}$ - timpul de funcționare virtuală până la defecțiune. Semnificația rezultatelor obținute constă în faptul că, prin monitorizarea dinamicii schimbărilor coeficientului statistic de disponibilitate a liniei aeriene pentru intervalele anterioare de funcționare normată, putem prezice timpul de aflare în stare deconectată al acesteia în următorul interval normat de exploatare.

Cuvinte-cheie: linii electrice aeriene, factor de disponibilitate tehnică, interval de predicție normat, timp virtual între defecțiuni, grad de confidență.

(C) Басманов В.Г.,

Холманских В.М. 2021 


\section{Особенности прогнозирования надёжности воздушных линий 6-10 кВ по статистическим данным об отказах и восстановлениях \\ Басманов В.Г., Холманских В.М. \\ Вятский государственный университет \\ Киров, Российская Федерация}

Аннотация. Целью работы является обоснование прогнозирования времени простоя воздушных линий (ВЛ) 6-10 кВ за нормируемый интервал её эксплуатации на основе сравнения статистических данных об отказах и восстановлениях за предыдущие интервалы с использованием функции готовности ВЛ, статистического коэффициента готовности, нормируемого интервала прогнозирования и допустимых значений коэффициента готовности на интервале прогнозирования. Для достижения поставленной цели ВЛ рассмотрена как объект многократного действия, её потоки отказов и восстановлений приняты простейшими пуассоновскими, а для теоретического анализа характера изменения коэффициента готовности использовались методы теорий вероятностей и массового обслуживания. Установлена высокая точность замены вероятности нахождения ВЛ в исправном состоянии на статистическое значение коэффициента готовности для установившегося режима эксплуатации. Ошибка вычислений при такой замене даже в диапазоне изменения значений коэффициента готовности за календарный год от 0,999 до 0,995 составляет от 0,018 до 0,369 \%. Наиболее существенным результатом является обоснование применения для прогнозирования времени простоя ВЛ нового удобного экспоненциального выражения её функции готовности на нормируемом интервале времени прогнозирования. В отличие от принятой в теории надежности функции готовности с двумя параметрами $T$ и $T_{\text {r.av }}$ (средних времён работы и восстановления) в предлагаемом выражении функции готовности ВЛ используется один параметр распределения $T_{v i r}$ - виртуальная наработка на отказ. Длина нормируемого интервала прогнозирования определяется заданной доверительной вероятностью статистических данных об отказах за предшествующий нормируемый интервал эксплуатации ВЛ. Значимость полученных результатов состоит в том, что, контролируя динамику изменения статистического коэффициента готовности ВЛ за предшествующие нормируемые интервалы времени можно предсказать её время простоя в предстоящем нормируемом интервале эксплуатации. Ключевые слова: воздушные линии, коэффициент технической готовности, нормируемый интервал прогнозирования, виртуальная наработка на отказ, доверительная вероятность.

\section{ВВЕДЕНИЕ}

Воздушные линии - это главные элементы систем электроснабжения. Протяженность ВЛ 6-10 кВ в России составляет более 1,5 млн. км - почти $45 \%$ от общей протяжённости линий электропередачи 0,4-110 кВ. Около 70\% всех отказов в электроснабжении приходится на сети данного класса напряжения [1].

ВЛ - наиболее повреждаемые элементы электрических сетей из-за территориальной протяженности и подверженности влиянию климатическим воздействиям. Их параметр потока отказов на порядок выше параметров потока отказов трансформаторов и выключателей [2]. Поэтому прогнозирование состояния ВЛ необходимо для поддержания бесперебойности электроснабжения.

В работах [3-12] приведены характерные причины повреждений и результаты анализа данных об отказах в воздушных линиях электропередач разных электросетей, например, в работе [6] приведены типичные причины неисправностей в воздушной линии по данным взятым из опросов различных мировых агентств. В работах [7-9] даны \begin{tabular}{llrr}
\multicolumn{1}{c}{ результаты } & анализа & \multicolumn{2}{c}{ данных } \\
неисправности & воздушных & и & кабельных \\
линий & среднего & напряжения & польской \\
национальной & энергосистемы. & В & [12]
\end{tabular} отмечено, что значительная часть ВЛ была введена в эксплуатацию 40-50 лет назад, что позволило избавиться от неэкономичных дорогостоящих маломощных электростанций и реализовать электроснабжение потребителей в городской и сельской местностях от сетей объединённых энергосистем. По данным [13, 14] срок службы проводов марок А и АС составляет 45 лет, а срок службы опор 30-35 лет. Понятно, что ресурс этих линий сейчас исчерпан, либо близок к нулю.

Расчеты по определению основных показателей надежности ВЛ (параметр потока отказов и среднее время восстановления), а также результаты их анализа представлены во многих работах, например, [7-10,11,15-26]. Для анализа проблемы в [7] был использован непараметрический метод анализа данных о надежности электросетей, а, например, в [8,9] изложен порядок количественной оценки надежности инженерных систем вероятностными методами. Подобные 
расчеты по количественной оценке надежности в электроэнергетических системах приводятся в [11,16,17-19] на основе метода статистического моделирования (метод Монте-Карло). В работе [24] предлагается целостный подход к проблеме надежности электрических систем. Рассматриваются, что крайне важно, в совокупности механизмы отказов, методы испытаний, анализ отказов, методы определения характеристик надежности и модели прогнозирования, для повышения безотказной работы целого ряда устройств.

Таким образом, вопрос поддержания надежности ВЛ весьма актуален. Несмотря на большое разнообразие в подходах и методах исследования надежности ВЛ в многочисленных вышеупомянутых работах, нами не найдено работ по прогнозированию времени простоя ВЛ, используя её функцию готовности. Только в работе [24] рассмотрены модели прогнозирования. Поэтому можно утверждать, что наш подход по выбору прогнозирования времени простоя воздушных линий (ВЛ) 6-10 кВ, с использованием нормированного интервала прогнозирования и функции готовности с одним параметром - виртуальной наработки на отказ, является оригинальным.

\section{І. ПОСТАНОВКА ЗАДАЧИ}

Целью данной работы является обоснование использования динамики статистического коэффициента готовности ВЛ за прошедшие периоды её эксплуатации для прогнозирования времени простоя в совокупности с вычисляемой функцией готовности за нормируемый интервал прогнозирования.

Для достижения поставленной цели необходимо рассмотреть ВЛ как объект многократного действия $\mathrm{c}$ простейшими пуассоновскими потоками отказов и восстановлений. Использовать комплексные статистические показатели надёжности, которые позволят одновременно оценивать свойства работоспособности и ремонтопригодности. Они должны легко определяться по статистическим данным об отказах. Известно, что такими показателями являются коэффициенты готовности $K_{\text {tesrf }}$ и простоя $K_{s}$, которые рассчитываются по итогам работы ВЛ за календарный год её эксплуатации или за другие фиксированные интервалы времени.

Для предсказания поведения $K_{\text {tesrf }}$ и $K_{s}$ на последующие годы работы ВЛ следует использовать теоретическую функцию готовности $K_{\text {tesrf }}(t)$ при этом требуется установить характер приближения $K_{t e s s f}(t)$ к статистическому значению $K_{\text {tesrf. }}$ Если этот процесс совершается быстро, то этим можно обосновать замену вероятностного значения $K_{\text {tesrf }}(t)$ на его статистическое значение $K_{\text {tesrf }}$ и выполнить прогнозирование.

Очень важно правильно определить длину нормируемого интервала времени, на котором предполагается выполнить прогноз простоя ВЛ с учётом принятой доверительной вероятности (достоверности) предшествующего статистического материала об отказах и восстановлениях ВЛ.

Необходимо обосновать применение для прогнозирования более простой формулы для функции готовности, исключив из известного выражения средние времена безотказной работы и восстановления. Должна быть доказана высокая точность предлагаемой формулы.

Результаты прогнозирования должны отвечать на вопрос: с какого прогнозируемого года эксплуатации время простоя ВЛ будет ниже заданного допустимого значения?

\section{II. МЕТОДЫ И АЛГОРИТМЫ РЕШЕНИЯ}

Прогнозирование времени простоя ВЛ является важным инструментом повышения надёжности ВЛ. Он позволяет принять правильное решение о первоочерёдности профилактических работ или замене «слабых» элементов при одновременной эксплуатации большого числа ВЛ, предопределяя надёжное электроснабжение потребителей электрической энергией.

На практике в электрических сетях организован сбор статистической информации о надёжности, но, к сожалению, эта важная информация об эксплуатационной надёжности используется не в полной мере.

\section{Особенности характеристик потока отказов ВЛ}

Воздушная линия (ВЛ) 6-10 кВ является объектом многократного действия, для которого характерно то, что в процесс его эксплуатации могут возникать отказы, на их 
отыскание и устранения требуется некоторые время. После чего эксплуатация ВЛ продолжается. Поэтому для характеристик надёжности ВЛ должны применяться её потоки отказов $\omega$ и восстановлений $\mu$.

Рассмотрим особенности характеристик потока отказов ВЛ на основе общих свойств и принятой классификации потоков случайных событий, которая дана в теории массового обслуживания $[27,28]$. ВЛ состоит из значительного числа одновременно работающих $\mathrm{n}$ элементов. Если произошёл отказ одного элемента, и он будет заменён исправным, то вероятнее всего следующий отказ будет не у самого «свежего» элемента, а у элемента с большим сроком эксплуатации в составе ВЛ. Поэтому взаимная зависимость отказов при любом законе вероятности безотказной работы будет тем слабее, чем больше элементов в составе ВЛ. На практике, когда $n$ элементов ВЛ достаточно велико, поток отказов можно считать ординарным без последействия. Такой поток полностью определяется параметром $\omega(t)$.

Типичный график $\omega(t)$ при наличии периода приработки имеет вид, представленный на рис. 1.

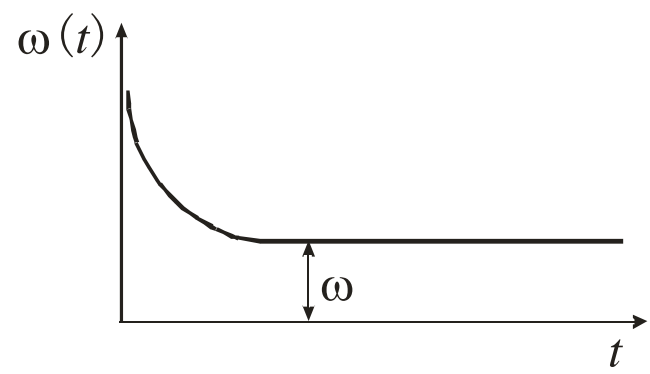

Рис. 1. Параметр поток отказов ВЛ в случае наличия периода приработки. ${ }^{1}$

В начальный период эксплуатации, который принято называть приработкой, функция $\omega(t)$ убывает, стремясь к $\omega(t)=$ const. При достижении к $\omega(t)=\omega=$ const поток отказов ВЛ становится простейшим и сохраняется в течение всего времени эксплуатации. Период износа отсутствует или не так ярко выражен, так как отказавшие элементы ВЛ всегда заменяются новыми или отремонтированными. Это подтверждается многочисленными статистическими исследованиями, приведенными в [29,30].

Необходимо отметить, что периода приработки может и не быть, если все элементы ВЛ имеют экспоненциальные законы распределения наработок на отказ, что следует из доказательства теоремы, приведённой в [31]. Суть теоремы в следующем: если у ординарного потока с ограниченным последействием все промежутки времени между соседними событиями распределены по одному и тому же экспоненциальному закону, то этот поток событий простейший с параметром

$$
\omega=n_{1} \lambda_{1}+n_{2} \lambda_{2}+\ldots+n_{k} \lambda_{k}
$$

где $n_{k}$ - количество элементов $k$-ой группы, входящих в состав ВЛ;

$\lambda_{k}$ - соответствующие интенсивности отказов элементов $k$-ой группы.

Для общего случая, когда ВЛ большой протяжённости состоит из $k$ групп по $n_{1}, n_{2}, \ldots, n_{k} \quad$ элементов с интенсивностью отказов соответственно $\lambda_{1}(t), \lambda_{2}(t), \ldots, \lambda_{k}(t)$, параметр $\omega(t)$ потока отказов ВЛ следует определять по уравнению

$$
\omega(t)=n_{1} \omega_{1}(t)+n_{2} \omega_{2}(t)+\ldots+n_{k} \omega_{k}(t),
$$

где $\omega_{k}(t)$ - параметр потока отказов, вызываемого работой одного элемента $k$-ой группы.

В [31] показано, что с ростом $t$ величины $\omega_{k}(t)$ стремятся к постоянным $\omega_{k}$, равным обратным значениям средних сроков службы элементов. Тогда из уравнения (2) следует, что параметр $\omega(t)$ потока отказов ВЛ имеет предел

$$
\omega=\frac{n_{1}}{T_{1}}+\frac{n_{2}}{T_{2}}+\ldots+\frac{n_{k}}{T_{k}}
$$

где $T_{1}, T_{2}, \ldots, T_{k}-$ средние сроки службы элементов ВЛ каждой $k$-й группы соответственно.

Уравнение (3) позволяет найти параметр потока отказов ВЛ после окончания периоды приработки по известным средним срокам службы всех элементов ВЛ для любых законов распределения длительности службы элементов.

Известно, что в случае простейшего потока событий для произвольного промежутка времени величиной $\tau$, среднее число отказов за это время будет

$$
m_{a v}=\omega \tau .
$$


Тогда среднее время безотказной работы $T$ определится по уравнению

$$
T=\tau / m_{a v} .
$$

Из (4) можно получить

$$
T=1 / \omega .
$$

Установив для простейшего потока событий показатели надёжности ВЛ - $\omega$ и $T$ можно определить вероятность $p(\tau)$ безотказной работы ВЛ от момента $t$ до момента $t+\tau$, где $t$ и $\tau$ произвольные

$$
p(\tau)=\exp (-\omega \tau)=\exp (-\tau / T) .
$$

Применение формулы (7) в практических расчётах надёжности затруднено из-за особенностей эксплуатации ВЛ. Покажем это на примере. Требуется определить Tсреднее время безотказной работы ВЛ, которое обеспечило бы $p(\tau)=0,999$ в течение 1 года. Условие задачи соответствует действительности (при коэффициенте готовности ВЛ за календарный год 0,999 суммарное время её простоя составит 8,76 часа).

Из формулы (7) определяем требуемую наработку на отказ ВЛ. $T=-8760 / \ln 0,999=$ 8755619 часов, что составляет около 1000 лет, т.е. полученный результат является нереальным из-за особенностей эксплуатации ВЛ, а именно, из-за постоянного «обновления» элементов ВЛ при их отказах, высоких требованиях к надёжности, продолжительных сроков эксплуатации ВЛ (десятки лет) [12-14] и ряда других причин.

Обоснование использования коэффичиента готовности для прогнозирования времени простоя ВЛ

В отличие от вероятности безотказной работы ВЛ (качественный единичный показатель) будут наиболее понятны и практически полезны такие комплексные показатели, как $K_{\text {tesrf }}$ и $K_{s}-$ соответственно коэффициенты готовности и простоя, которые могут рассчитываться по итогам работы ВЛ за календарный год её эксплуатации или за другие фиксированные интервалы времени.

Приведём известные выражения для их расчёта и рассмотрим характер изменения этих показателей надёжности в процессе длительной эксплуатации ВЛ.

Как известно $K_{t e s i f}$ и $K_{s}$ применяют, когда требуется оценить одновременно свойства работоспособности и ремонтопригодности. При расчётах $K_{\text {tesrf }}$ и $K_{s}$ используются две важные статистические величины суммарное время исправной работы $t_{t w t}$ и суммарное время вынужденных простоев (восстановлений) $t_{r}$, взятые за один и тот же календарный срок.

$$
t_{t w t}=\sum_{1}^{n} t_{t w t i}, t_{r}=\sum_{1}^{n} t_{r i},
$$

где $t_{t w t i}$ и $t_{r i}-i$-е интервалы времени исправной работы и восстановления ВЛ после $i$-го отказа;

\section{$\mathrm{n}$ - число отказов.}

По известным $t_{t w t}$ и $t_{r}$ определяются $K_{t e s r f}$. и $K_{s}$.

$$
\begin{gathered}
K_{\text {tessf }}=\frac{t_{t w t}}{t_{t w t}+t_{r}}, \\
K_{s}=\frac{t_{r}}{t_{t w t}+t_{r}} .
\end{gathered}
$$

От соотношения величин $t_{t w t}$ и $t_{r}$ зависит величины $K_{\text {tesrf }}$ и $K_{s}$.

Разделив числитель и знаменатель выражений (9) и (10) на число отказов $n$, произошедших за рассматриваемое время, получим следующие выражения для $K_{t e s i f}$ и $K_{s}$

$$
\begin{aligned}
K_{\text {tessif }} & =\frac{T}{T+T_{r . a v}}, \\
K_{s} & =\frac{T_{r . a v}}{T+T_{r . a v}},
\end{aligned}
$$

где $T$ и $T_{\text {r.av }}$ - соответственно среднее время безотказной работы и восстановления ВЛ. В теории надежности $T$ имеет название средняя наработка на отказ. Будем считать, что $T_{\text {r.av }}$ соответствует времени простоя ВЛ, т.е. включает в себя время отыскание повреждения, демонтаж отказавшего элемента и монтаж исправного.

Физический смысл коэффициента готовности ВЛ это вероятность застать ВЛ в исправном состоянии, когда параметры её 
потока отказов $\omega$ и восстановлений $\mu$ есть величины постоянные, т.е. соответственно равны интенсивностям отказов и восстановлений.

Рассмотрим характер изменения $K_{\text {tesrf }}$ в процессе эксплуатации ВЛ, используя теоретический материал, изложенный в [31]. Уравнения (11) и (12) справедливы для установившегося режима работы. В общем случае работа ВЛ соответствует следующим условиям. ВЛ начинает работать в момент $t=0$. Отказы и восстановления образуют простейшие потоки событий с параметрами соответственно $\omega=1 / T$ и $\mu=1 / T_{\text {r.av }}$.

В произвольный момент времени ВЛ может находиться в одном из двух состояний: или в исправном, вероятность состояния которого обозначим как $p_{0}(t)$, или в состоянии ремонта с вероятностью $p_{1}(t)$. Простои ВЛ, не связанные с ремонтом, не рассматриваем.

Для этого момента справедливо уравнение

$$
p_{0}(t)+p_{1}(t)=1
$$

Принимаем начальные условия

$$
p_{0}(0)=1, p_{1}(0)=0 \text {. }
$$

Рассмотрим малый промежуток времени от момента $t$ до момента $t+\Delta t$. Вычислим вероятность того, что в конце этого промежутка времени ВЛ будет исправна. Здесь могут возникнуть два возможных состояния:

1) в момент $t$ ВЛ была исправна (вероятность этого состояния $p_{0}(t)$, а за время $\Delta t$ отказов не было (вероятность этого состояния равна $1-\omega \Delta t$.

2) в момент $t$ ВЛ была в ремонте (вероятность этого состояния $\left.p_{1}(t)\right)$, а за время $\Delta t$ восстановилась (вероятность этого состояния равна $\mu \Delta t)$.

Из этого следует, что

$$
p_{0}(t+\Delta t)=p_{0}(t)(1-\omega \Delta t)+p_{1}(t) \mu \Delta t .
$$

Перенесём $p_{0}(t)$ в левую часть, разделим на $\Delta t$ и перейдём к пределу при . В результате получим дифференциальное уравнение

$$
\frac{d p_{0}}{d t}=-\omega p_{0}(t)+\mu p_{1}(t)
$$

Из уравнений (16) и (13) найдём

$$
\frac{d p_{0}}{d t}=\mu-(\omega+\mu) p_{0}(t)
$$

Решение этого уравнения при начальных условиях (14) имеет вид

$$
p_{0}(t)=\frac{\mu}{\omega+\mu}+\frac{\omega}{\omega+\mu} \exp [-(\mu+\omega) t]
$$

Преобразуем уравнение (18) с учётом (11), (12) и равенств: $K_{\text {tesrf }}+K_{s}=1, \quad \omega=1 / T \quad$ и $\mu=1 / T_{r . a v}$ к окончательному виду

$$
p_{0}(t)=K_{t e s i f}+\left(1-K_{t e s i f}\right) \exp \left(-t / T \cdot T_{r . a v}\right)
$$

Необходимо отметить, что аналогичный результат можно получить, используя математический аппарат для анализа надёжности восстанавливаемых СMO, разработанный на основе марковской модели с дискретным множеством состояний и непрерывным временем [32-34]. Потоки, переводящие систему из состояния в состояние, должны быть пуассоновскими, а законы распределения наработки до отказа и времени восстановления экспоненциальными. Структуры системы изображается в виде графов состояний с прямыми (отказ) и обратными (ремонт) переходами.

Рассматриваются марковские процессы, у которых для любого момента времени вероятность каждого состояния системы в будущем зависит только от состояния в настоящий момент, и не зависит от того, каким образом система пришла в это состояние (потоки без последействия).

Использование марковских случайных процессов в системах электроснабжения подтверждается практикой их эксплуатации [35].

Характер приближения вероятностного значения $K_{\text {tesrff }}(t)$ к его статистическому значению

Из (19) видно, что при $t=0$ ВЛ находится в исправном состоянии с вероятностью 
$p_{0}(t)=1$. По мере её эксплуатации $p_{0}(t) \rightarrow K_{\text {tesrf }}$. Для прогнозирования надёжности ВЛ по статистическим данным очень важно установить характер приближения к $K_{\text {tesrf. }}$ Если этот процесс совершается быстро, то можно заменить вероятностное значение $K_{\text {tesrf }}(t)$ на его статистическое значение $K_{t e s r f}$. В теории надежности $K_{\text {tesrf }}(t)=p_{0}(t) \quad$ называется функцией готовности и вычисляется по выражению (20).

$$
K_{\text {tesif }}(t)=K_{\text {tesrf }}+\left(1-K_{\text {tesif }}\right) \exp \left(\frac{-t}{T \cdot T_{\text {r.av }}}\right) .
$$

В [36] представлен один из вариантов анализа точности такой замены.

В тех случаях, когда $t_{i} \leq-T \ln K_{t e s f}$ вероятность нахождения ВЛ в исправном состоянии можно считать равной соответствующему значению вероятности её безотказной работы за период $\left(0, t_{i}\right)$.

Ошибка вычислений от такой замены при $K_{\text {tesrf }}>0,9$ составит

$$
\Delta K_{\text {tesif }}(t) \approx\left(1-K_{\text {tesif }}\right) K_{\text {tesrf }} \frac{K_{\text {tessf }}}{1-K_{\text {tessf }}} 100 \%
$$

Рассмотрим реальный диапазон допустимых значений $K_{\text {tesrf }}$ ВЛ за календарный год (8760 часов) от 0,999 до 0,995, что соответствует диапазону вынужденных простоев в течение года от 4,38 до 43,8 часа. Тогда ошибки вычислений по (21) будут в пределах от 0,018 до 0,369 \%. На практике к надёжности ВЛ предъявляются более жёсткие требованиях от 0,9999 до 0,9995, тогда ошибки вычислений будут ещё меньше. Таким образом, с учётом высокой надёжности ВЛ ошибка при замене статистического коэффициента готовности на вероятность нахождения ВЛ в исправном состоянии ничтожно мала.

В [30] сделан аналогичный вывод. Доказывается, что даже при $K_{\text {tesrf }}=0,9 \quad p_{0}(t)$ быстро стремится к постоянной величине $K_{\text {tesif }}=0,9$. Этим важным обстоятельством воспользуемся для прогнозирования времени простоя ВЛ за предстоящее время её эксплуатации по имеющейся статистике отказов.
Обоснование использования для прогнозирования функции готовности ВЛ, выраженной через виртуальную наработку на отказ

C учётом вышеизложенного, прогнозирование $K_{t e s r f}$ за последующие годовые интервалы работы ВЛ можно выполнить по (20), используя статистические значения $T$ и $T_{\text {r.av }}$, за предыдущий период с момента эксплуатации. Но при таком подходе к прогнозированию его точность будет под сомнением по следующим обстоятельствам. Фактическое время эксплуатации ВЛ может достигнуть нескольких десятков лет [12-14], за которое большинство её элементов многократно заменяться новыми или отремонтированными. Поэтому $K_{\text {tesrf }}$ за каждый прошедший год работы будет иметь случайный характер из-за случайного изменения $T$ и $T_{\text {r.av }}$.

Точность прогноза $T$ и $T_{\text {r.av }}$, увеличиться, если использовать их средние статистические значения, рассчитанные на нескольких прошедших одинаковых интервалах времени, длины которых определяются заданным значением доверительной вероятности результатов расчёта $T$ и $T_{\text {r.av }}$ на этих интервалах.

В выражении (20), присутствует параметр $T_{\text {r.av }}$, что не всегда удобно. Можно в неявном виде исключить этот параметр, если на вышеназванных интервалах расчёт выполнять не по (20), а по предлагаемой нами формуле

$$
K_{\text {tesrf }}(t)=e^{-t / T_{\text {vir }}}
$$

где $T_{v i r}$ - виртуальная наработка на отказ.

Виртуальная наработка на отказ - это среднее время безотказной работы ВЛ, при котором её $K_{t e s r f}$ равен вероятности застать систему в исправном состоянии $P(t)$ за выбранный интервал прогнозирования от 0 до $t$, считая точкой отсчёта экспоненты (22) начало интервала прогнозирования.

Сравним результаты вычислений $K_{t e s r f}$ за календарный год работы по формулам (20) и (22) для ВЛ одинаковой длины, если статистическое значение $K_{\text {tesrf }}$ за календарный год составило 0,9999.

Найдем $T_{v i r}$.

$$
T_{v i r}=-t / \ln 0,9999=10000 \text { лет }
$$


Из определения $T_{v i r}$ следует, что $K_{t e s r}$, вычисленный по (22) равен

$$
K_{\text {tessf }}(1)=e^{-1 / 10000}=0,9999
$$

По (20) определим значение $K_{\text {tesrf }}(1)$, которое сравним с 0,9999 .

$K_{\text {tessf }}(1)=0,9999+(1-0,9999) \exp [-10000 /(10000$.

$\cdot 0,0001)]=0,9999368$

где $T_{r . a v}=\left(1-K_{t e s s f}\right) \cdot 1=0,0001$ год.

Таким образом, ошибка составит менее $0,004 \%$

Определение длины нормируемого интервала прогнозирования

Очень важно правильно определить длину интервала времени, на котором предполагается выполнить прогноз простоя ВЛ. Дадим этому интервалу название нормируемый исходный интервал прогнозирования (далее интервал прогнозирования) и обозначим его $\Delta t_{\text {norm.in }}$.

Если замена текущих значений $P(t)$ на $K_{\text {tesrf, }}$ как показано выше, не вызывает больших ошибок, то выбор длины интервала прогнозирования, напротив, существенно влияет на точность прогноза. Дело в том, что реальное значение среднего времени безотказной работы ВЛ определяется либо по справочным данным, либо по «свежим» статистическим данным об отказах и восстановлениях. В справочных таблицах [37] приведены средние значения интенсивностей или параметров потока отказов, которые не всегда соответствуют реальным значениям. Можно утверждать, что чем больше продолжительность эксплуатации ВЛ, тем меньше её средние наработки на отказ, вычисленные по статистическим данным за последующие фиксированные интервалы времени. Но на практике может встретиться и противоположная ситуация, например, у ВЛ, капитально отремонтированной с многочисленными заменами морально устаревших элементов на современные. Нужно отметить, что такая ситуация встречается редко. Точность статистических оценок среднего времени безотказной работы зависит от продолжительности сбора информации. Чем больше время сбора информации, тем больше её объем, тем точнее статистические оценки среднего времени безотказной работы и достовернее прогноз. Поэтому величину интервала прогнозирования целесообразно согласовать с такой продолжительностью сбора статистических данных, которая бы обеспечила расчёты средних времён безотказной работы ВЛ в интервалах прогнозирования с требуемой доверительной вероятностью $\alpha$. Воспользуемся выражением (10), приведённым в [38] для определения объёма испытаний в случае распределения Пуассона.

$$
n t_{i}=\frac{m}{\lambda_{0} r_{3}},
$$

где $n$ - число испытываемых ВЛ одного назначения;

$t_{i}$ - время испытания;

$\mathrm{m}$ - число отказов за время испытания;

$\lambda_{0}$ - ожидаемое значение интенсивности отказов;

$r_{3}$ - коэффициент, используемый при определении доверительных границ для распределения Пуассона.

В нашем случае $n=1$, тогда (23) будет иметь вид

$$
\Delta t_{\text {norm.in }}=\frac{m}{\lambda_{0} r_{3}} .
$$

\section{Порядок прогнозирования}

По условиям требования к надёжности ВЛ задаётся допустимый интервал изменения её коэффициента готовности

Исходное значение $T_{\text {vir in }}$ рассчитывается по формуле

$$
T_{\text {vir in }}=-\Delta t_{\text {norm.in }} / \ln K_{\text {tessf min }} .
$$

Рассчитывается по статистическим данным об отказах и восстановлениях ВЛ за прошедшие годы средние времена $T_{1}$ и $T_{2}$ безотказной работы за 2 последних интервала эксплуатации. Тогда параметры потоков отказов за эти интервалы определятся как $\omega_{1}=1 / T_{1}, \omega_{2}=1 / T_{2}$.

Для определения времени простоя ВЛ на первом интервале прогнозирования строится экспонента (22) при скорректированном значении $T_{\text {vir cor } 1}$, задаваясь временем от 0 до $0+\Delta t_{\text {norm.in }}$. Где $T_{\text {vir cor } 1}$ рассчитывается по формуле

$$
T_{v i r \text { cor } 1}=T_{v i r} \cdot\left(\omega_{1} / \omega_{2}\right) .
$$

Для определения времени простоя ВЛ на прогнозируемом интервале выразим 
полученное по (22) значение $K_{\text {tesrf }}$ через (11) и рассчитаем время простоя на первом предстоящем интервале прогнозирования для любого интересующего нас момента времени в пределах интервала. После завершения эксплуатации ВЛ на первом прогнозируемом интервале обрабатываются статистические данные об отказах и восстановлениях и вычисляется параметр потока отказов $\omega_{3}=1 / T_{3}$. Находится новое скорректированное значение $T_{\text {vir cor } 2}=T_{\text {vir cor } 1} \cdot\left(\omega_{2} / \omega_{3}\right)$, строится по (22) новая экспонента при новых скорректированных значениях $T_{\text {vir cor } 2}$ и $\Delta t_{\text {norm.cor }}$, задаваясь временем от 0 до $0+\Delta t_{\text {norm.cor }}$. Для определения времени простоя на втором интервале прогнозирования расчёты повторяются и так далее до интересующего нас очередного интервала прогнозирования.
Таким образом, для предлагаемого метода прогнозирования времени простоя ВЛ в предстоящем интервале прогнозирования необходимо иметь обновлённые статистические данные по средним временам безотказной работы ВЛ в двух предыдущих интервалах прогнозирования. В современных условиях это не представляет особых трудностей, так как во всех электрических сетях в обязательном порядке предусмотрен сбор статистической информации об отказах и восстановлениях ВЛ.

\section{III. РЕЗУЛЬТАТЫ И ИХ ОБСУЖДЕНИЕ}

Покажем на реальном примере прогнозирование времени простоя ВЛ на нормируемом интервале.

Результаты расчётов представим в виде итоговой таблицы 1 и графика (рис. 2).

Таблица $1^{2}$.

Результаты расчётов прогнозируемых показателей надёжности ВЛ 6-10 кВ $L=10$ км в нормируемом интервале прогнозирования 6 лет. ${ }^{3}$

\begin{tabular}{|c|c|c|c|c|}
\hline $\begin{array}{l}\text { Годы работы ВЛ } \\
\text { в нормируемом } \\
\text { интервале } \\
\text { прогнозирования } \\
\text { (OL years of } \\
\text { operation } \\
\text { normalized on } \\
\text { forecasting } \\
\text { interval), } t_{i}\end{array}$ & $\begin{array}{l}\text { Прогноз } \\
\text { коэффициент } \\
\text { a готовности } \\
\text { (Availability } \\
\text { coefficient } \\
\text { forecasting), } \\
K_{\text {tesrf } i}\end{array}$ & $\begin{array}{l}\text { Прогноз } \\
\text { коэффициента } \\
\text { простоя } \\
\text { (Unavailability } \\
\text { coefficient } \\
\text { forecasting), } \\
K_{s i}=1-K_{\text {tesrf } i}\end{array}$ & $\begin{array}{l}\text { Прогноз времени } \\
\text { простоя ВЛ по годам } \\
\text { эксплуатации } \\
\text { нарастающим итогом в } \\
\text { часах } \\
\text { (Forecasting of OL idle } \\
\text { hours in operation time } \\
\text { increasing for years), }\end{array}$ & $\begin{array}{l}\text { Прогноз времени } \\
\text { простоя ВЛ по годам } \\
\text { эксплуатации в часах } \\
\text { (Forecasting of OL } \\
\text { idle hours in operation } \\
\text { time for years), } \\
\left(K_{s i+1}-K_{s i}\right) \cdot 8760\end{array}$ \\
\hline 1 & 0,99981 & 0,00019 & 1,66 & 1,66 \\
\hline 2 & 0,99966 & 0,00034 & 2,98 & 1,32 \\
\hline 3 & 0,99950 & 0,00050 & 4,38 & 1,40 \\
\hline 4 & 0,99933 & 0,00067 & 5,87 & 1,49 \\
\hline 5 & 0,99916 & 0,00084 & 7,36 & 1,49 \\
\hline 6 & 0,99900 & 0,00100 & 8,76 & 1,40 \\
\hline
\end{tabular}

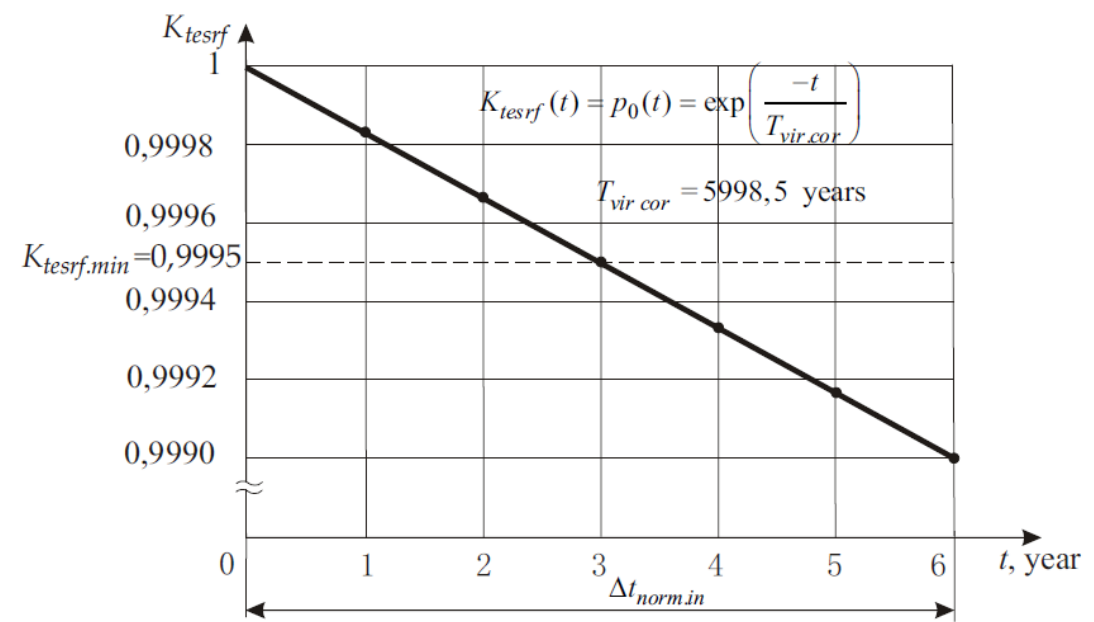

Рис. 2. График изменения $K_{t e s i f}(t)$ ВЛ 6-10 кВ ( $L=10$ км) в нормируемом интервале прогнозирования. ${ }^{4}$ 
Выполнить прогноз времени простоя одноцепной ВЛ 6-10 кВ длиной $L=10$ км. Известно, что параметры потока отказов по статистическим данным за последние 2 шестилетних интервала времени равны соответственно $\omega_{1}=1$ (1/год), $\omega_{2}=2$ (1/год). В пересчёте на 1 км длины ВЛ это составит соответственно $\omega_{1}=0,1$ (1/год) и $\omega_{2}=0,2$ (1/год). Показатель вполне реальный, так как табличное значение параметра аналогичной ВЛ равно 0,25 (1/год) на 1 км [37].

Требуется дать прогноз времени простоя ВЛ в первом интервале прогнозирования на каждый год эксплуатации. Длину интервала прогнозирования рассчитать с доверительной вероятностью $\alpha=0,8$. Допустимый интервал изменения коэффициента готовности ВЛ на интервале прогнозирования принять равным

Решение

1. По (25) рассчитываем исходный интервал прогнозирования.

В нашем примере $\lambda_{1}=\omega_{1}=1 \quad(1 /$ год $)$, $\lambda_{2}=\omega_{2}=2(1 /$ год $)$

Из таблицы П19 [31] для $\alpha=0,8, m=10$ находим $r_{3}=0,8$. Выбираем $\lambda_{0}=\lambda_{2}=\omega_{2}=2$ (1/год)

$$
\Delta t_{\text {norm.in }}=\frac{10}{2 \cdot 0,8}=6,25 \text { года. }
$$

Принимаем $\Delta t_{\text {norm.in }}=6$ лет.

2. Определяем исходное значение $T_{v i r}=-6 / \ln 0,9995=11997$ лет. 3. По
рассчитываем $T_{\text {vir cor } 1}=T_{\text {vir }} \cdot\left(\omega_{1} / \omega_{2}\right)=11997 \cdot(1 / 2)=5998,5$ года.

4. По (22) выполняем построение экспоненты на первом интервале прогнозирования от $t_{1}=0$ до $t_{2}=6$ лет при $T_{\text {vir cor } 1}=5998,5$ года.

5. По вышеизложенному порядку прогнозирования определяем время простоя ВЛ в каждом из шести предстоящих годах эксплуатации.

6. По характеру изменению коэффициента готовности ВЛ в течение 6-летнего прогнозируемого интервала делаем соответствующие выводы.

Из таблицы 1 видно, что начиная с 4-го прогнозируемого года эксплуатации, время простоя ВЛ будет больше нижнего допустимого значения 1,4 часа, что вызовет изменение коэффициента готовности ВЛ ниже требуемого по заданию значения 0,9995 .

\section{IV. ВЫВОДЫ}

1. Подтверждено, что для высоконадежных ВЛ можно заменить текущие вероятностные значения $K_{\text {tessf }}(t)$ на статистические для установившегося режима и выполнить прогноз времени простоя ВЛ.

2. Для прогнозирования времени простоя ВЛ предложено новое простое экспоненциальное выражение для функции готовности ВЛ с одним параметром распределения $T_{v i r}$ (виртуальная наработка на отказ).

Подтверждена его высокая точность в сравнении с принятым в литературе выражением функции готовности с двумя параметрами распределения - средняя наработка на отказ $T$ и среднее время восстановления $T_{r . a v}$.

3. Предложен новый метод прогнозирования времени простоя ВЛ на нормируемом интервале её эксплуатации на основе сравнения статистических данных об отказах и восстановлениях ВЛ за 2 прошедших нормируемых интервала прогнозирования. Метод может быть использован не только для прогнозирования времени простоя ВЛ, но и для прогнозирования других комплексных показателей надёжности при наличии достаточной базы статистических данных об отказах, восстановлениях и технических обслуживаниях.

5. Использование предлагаемого метода позволяет объективно оценить и сравнить поведение нескольких ВЛ в последующие годы эксплуатации и заранее запланировать первоочередной комплекс ремонтов и технических обслуживаний ВЛ, более склонных по прогнозу к отказам.

6. Данный метод прогнозирования может быть использован не только для ВЛ 6-10 кВ, но и для других объектов систем электроснабжения многократного действия: ВЛ любого напряжения, кабельной линии, трансформаторной подстанции, системы электроснабжения промышленного предприятия. 


\section{APPENDIX 1 (ПРИЛОЖЕНИЕ 1)}

${ }^{1}$ Fig. 1. Parameter of OL failure flow in the presence of the non failure operating time period.

${ }^{2,3}$ Table 2. Results of calculation the forecasting indices of $6-10 \mathrm{kV}$. ( $\mathrm{L}=10 \mathrm{~km})$ OL reliability on the normalized forecasting 6-year interval.

${ }^{4}$ Fig. 2. Graph of change in $K_{\text {tesif }}(t) \quad 6-10 \mathrm{kV}$. (L=10 $\mathrm{km}) \mathrm{OL}$ on forecasting normalized interval.

\section{Литература (References)}

[1] Vorotnitsky V.V., Buzin S.A. Reklouzer novyiuroven' avtomatizatsii i upravleniyaVL 6-10 $\mathrm{kV}$ [Recloser - a new level of automation and control of 6-10 kV overhead lines]. Novostielectrotekhniki - Electrical Engineering News, 2005, no.3. (In Russian). Available at: http://www.news.elteh.ru/arh/2005/33/11.php (accessed 10.09.2020).

[2] Faibisovich D.L. Spravochnik po proektirovaniyu elektricheskikh setei [Reference book on the design of electrical networks]. 4th ed., Rev. and add. Moscow: Publishing house NTs ENAS, 2012. 352 p. (In Russian).

[3] .Barg I.G., Edelman V.I. Vozdushnye linii elektroperedachi: voprosy ekspluatatsii $i$ nadezh$n o s t i$ [Overhead lines: Issues of operation and reliability]. Moscow, Energoatomizdat, 1985. 248 p. (In Russian).

[4] Barg I.G. Nadezhnost' VL 0,4-20 kV sel'skokhozyajstvennogo naznacheniya [Reliability of overhead lines $0.4-20 \mathrm{kV}$ for agricultural purposes]. Energeticheskoe stroitel'stvo - Energy construction, 1992, no. 4. pp. 19-21. (In Russian).

[5] Zhulev A.N., Barg I.G. O vliyanii defektov, dopushchennykh pri sooruzhenii VL, na ikh nadezhnosti [On the influence of defects during the construction of overhead lines on their reliability]. Energeticheskoe stroitel'stvo - Energy construction, 1992, no. 7 pp. 33-38. (In Russian).

[6] Li Wang. The Fault Causes of Overhead Lines in Distribution Network. MATEC Web of Conferences. vol.61, 2016. The International Seminar on Applied Physics, Optoelectronics and Photonics (APOP 2016). Shanghai, China, 2016 doi: 10.1051/matecconf/20166102017.

[7] Kornatka M. Analysis of the exploitation failure rate in Polish MV networks. Eksploatacja $i$ Niezawodnosc - Maintenance and Reliability 2018, no 20 (3), pp. 413-419. doi: 10.17531/ein.2018.3.9.

[8] Chojnacki A.Ł. Chojnacka K.J. Niezawodność elektroenergetycznych sieci dystrybucyjnych [Reliability of power distribution networks]. Monografie, Studia, Rozprawy NR M100. Politechnika Świętokrzyska. Kielce 2018. p.467.

[9] Chojnacki A.Ł. Analiza niezawodności eksploatacyjnej elektroenergetycznych sieci dystrybucyjnych, Rozprawa habilitacyjna, Kielce 2013.

[10] Doletskaya L.I., Solopov R.V., Kavchenkov V.P., Andreenkov E.S. Analyzing the Reliability of Mechanical Parts in $10 \mathrm{kV}$ Aerial Transmission Lines under Ice-Coating and Wind Effects in View of Their Design Features. Mechanics, resource and diagnostics of materials and structures (MRDMS-2017). AIP Conference Proceedings. vol. 1915. 2017. doi: 10.1063/1.5017355

[11] Clements D., Mancarella P. Systemic modelling and integrated assessment of asset management strategies and staff constraints on distribution network reliability. Electric Power Systems Research. vol. 155. 2018. pp. 164-171. doi: 10.1016/j.epsr.2017.09.029.

[12] Andrievsky V.N., Golovanov A.T., Zelichenko A.S. Ekspluatatsiya vozdushnykh linii elektroperedachi [Operation of overhead power lines]. 3rd ed., revised. and add. Moscow, Energiya, 1976. 616 p. (In Russian).

[13] GOST 839-2019 Provoda neizolirovannye dlya vozdushnykh linii elektroperedachi. Tekhnicheskie usloviya[State Standart839-2019 Non-insulated conductors for overhead power lines. Specifications].Moscow, Standartinform Publ., 2019. 43 p.

[14] STO 56947007-29.240.01.053-2010 Metodicheskie ukazaniya po provedeniyu periodicheskogo tekhnicheskogo osvidetel'stvovaniya vozdushnykh linii elektroperedachi ENES [STO 5694700729.240.01.053-2010 Guidelines for conducting periodic technical inspection of UNEG overhead lines. Organization standard. JSC "FGC UES"]. (In Russian). Available at: https://normativ.su/catalog/standart/1001/319013/ (accessed 05.09.2021).

[15] Kutsenko G.F., Puhalskaya O. Yu. Osnovnye pokazateli nadezhnosti LEP 6-10 kV [Main indicators of reliability of $6-10 \mathrm{kV}$ overhead lines]. Energetika. Izvestiya vysshikh uchebnykh zavedenii $i$ energeticheskikh ob"edinenii SNG Energy. Proceedings of higher educational institutions and energy associations of the CIS, 2006, no. 6 pp.20-23. (In Russian).

[16] Billinton R., Allan R.N. Reliability Evaluation of Power Systems. Springer Science \& Business Media, 2013. p. 514.

[17] Goel L. Monte Carlo simulation-based reliability studies of a distribution test system. Electric Power Systems Research, vol.54, no. 1, 2000, pp. 55-65. doi: 10.1016/S0378-7796(99)00066-8.

[18] Heydt G.T., Graf T.J. Distribution system reliability evaluation using enhanced samples in a Monte Carlo approach. Transactions on Power Systems, vol. 25, no. 4, 2010, pp. 2006-2008, doi: 10.1109/TPWRS.2010.2045929. 
[19] Billinton R., Li W. Reliability Assessment of Electric Power Systems Using Monte Carlo Methods. Springer Science \& Business Media, 2013. p. 352.

[20] Alvarez-Alvarado M.S., Jayaweera D. Bathtub curve as a Markovian process to describe the reliability of repairable components. Generation, Transmission \& Distribution, vol. 12, no.21, 2018. pp. 5683-5689. doi: 10.1049/iet-gtd.2018.5505.

[21] Heylen E., Ovaere M., Proost S., Deconinck G., Van Hertem D. A multi-dimensional analysis of reliability criteria: From deterministic $N-1$ to a probabilistic approach. Electric Power Systems Research. vol. 167. 2019. pp. 290-300. doi: 10.1016/j.epsr.2018.11.011

[22] Kolcun Michal, Kornatka Miroslaw, Gawlak Anna, Conka Zsolt. Benchmarking the reliability of medium-voltage lines. Journal of ELECTRICAL ENGINEERING, vol. 68, no. 3, 2017. pp. 212-215.

[23] Grakowski L., Chojnacki A.L., Gebczyk K., Banasik K. Statistical Analysis and Modeling of the Reliability of Overhead Low Voltage Lines. Przeglad elektrotechniczny. vol. 95, no. 12, 2019. pp. 261-264. doi: 10.15199/48.2019.12.59

[24] Swingler J. (Ed.) Reliability characterisation of electrical and electronic systems. Woodhead Publishing, 2015. $274 \mathrm{p}$.

[25] Iešmantas T., Alzbutas R. Bayesian spatial reliability model for power transmission network lines. Electric Power Systems Research. vol. 173. $2019 . \quad$ pp. 214-219. doi: 10.1016/j.epsr.2019.04.014.

[26] Iešmantas T., Alzbutas R. Bayesian Methods for Analysis of Electric Grid Outages. Safety and Reliability. vol. 33, no. 4. 2013. pp. 12-23. doi: 10.1080/09617353.2013.11690978.

[27] Gnedenko B.V., Kovalenko I.N. Vvedenie v teoriyu massovogo obsluzhivaniya [On the Theory of Mass Service]. Moscow, Nauka, 1966, p. 301. (In Russian).

[28] Khinchin A.Y. Mathematical Methods in the Theory of Queuing. Dover Publications. 2013. $128 \mathrm{p}$.

[29] Barlow R.E., Proschan F. Mathematical Theory of Reliability. Philadelphia: SIAM, 1996. 258 p.

[30] Shor Ya.B. Statisticheskie metody analiza $i$ kontrolya kachestva i nadezhnosti: ucheb. dlya

\section{About authors.}

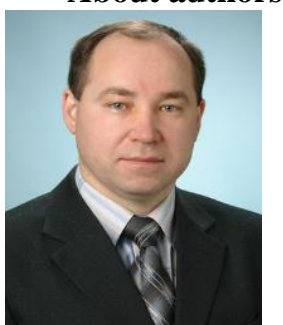

Басманов Владислав Геннадьевич, к.т.н., доцент, заведующий кафедрой «Электроснабжение» ВятГУ. Область научных интересов: диагностика электрооборудования, надежность электроснабжения, энергосбережение. E-mail: basmanov@vyatsu.ru vuzov [Statistical methods of analysis and quality control and reliability: textbook for universities]. Moscow, Gosenergoizdat, 1962. 552 p. (In Russian).

[31] Hooke J.B. Teoriya nadezhnosti v elektroenergetike [The theory of reliability in the electricity industry]. Leningrad, Energoatomizdat, 1990. 206 p. (In Russian).

[32] ] Endreni D. Modelirovanie pri raschetakh nadezhnosti $v$ elektroenergeticheskikh sistemakh [Modeling during Reliability Calculation in Electric Power Systems]. / D. Endreni; trans. from Eng. by B. N. Kazak; ed. Yu. N. Rudenko. - Moscow, Energoatomizdat, 1983. 336 p. (In Russian).

[33] Ryabinin I.A., Kireev Yu.N. Nadezhnost' sudovykh elektroenergeticheskikh sistem i sudovogo elektrooborudovaniya [Reliability of Ship Electric Power Systems and Ship Electric Equipment]. Leningrad: Sudostroenie, 1974, p. 264. (In Russian).

[34] Fokin Yu. A., Tufanov V.A. Otsenka nadezhnosti sistem elektrocyabzheniya [Assessment of the reliability of power supply systems]. Moscow, Energoizdat, 1981, p. 224. (In Russian).

[35] Druzhinin G.V. Nadezhnost' sistem avtomatiki [Automatic Systems Reliability]. Moscow, Energy, 1976, p. 528. (In Russian).

[36] Teoriya nadezhnosti radioelektronnykh sistem $v$ primerakh $i$ zadachakh. Uchebnoe posobie dlya studentov radiotekhnicheskikh spetsial'nostey vyzov. [Reliability Theory in Radio Electronic Systems Using Examples and Problems. Training Manual for Students of Radio Technical Colleges]. Ed. G.V. Druzhinin, Moscow, Energy, 1976, p. 448. (In Russian).

[37] Gul V.I., Nizhevsky V.I., Khomenko I.V., Shevchenko S.Yu., Chevychelov V.A. Koordinatsiya izolyatsii i perenapryazheniya $v$ elektricheskikh vysokovol'tnykh setyakh [Coordination of insulation and overvoltage in electrical highvoltage networks]. Kharkov, EDNA, 2009, p. 270. (In Russian).

[38]Shor Ya.B., Kuz'min F.I. Tablitsy dlya analiza $i$ kontrolya nadezhnosti [Tables for Analysis and Reliability Control]. Moscow, Sovetskoe Radio Publ. 1968, p. 288. (In Russian).

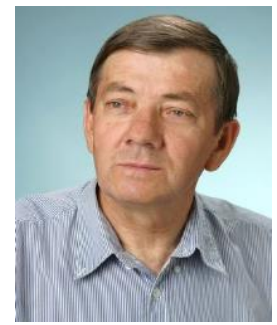

Холманских Валерий Михайлович, к.т.н., доцент, доцент кафедры «Электроснабжение» ВятГУ. Область научных интересов: надежность электроснабжения, энергосбережение.

E-mail:

vm_holmanskih@vyatsu.ru 